\title{
Crop Surveillance Demonstration Using a Near-Daily MODIS Derived Vegetation Index Time Series
}

\author{
Rodney McKellip \\ NASA Applied Sciences Directorate \\ John C. Stennis Space Center, MS 39529 USA \\ rodney.mckellip@ssc.nasa.gov
}

\author{
Robert E. Ryan and Slawomir Blonski \\ Science Systems \& Applications, Inc. \\ John C. Stennis Space Center, MS 39529 USA
}

\author{
Don Prados \\ Computer Sciences Corporation \\ John C. Stennis Space Center, MS USA 39529
}

\begin{abstract}
Effective response to crop disease outbreaks requires rapid identification and diagnosis of an event. A near-daily vegetation index product, such as a Normalized Difference Vegetation Index (NDVI), at moderate spatial resolution may serve as a good method for monitoring quick-acting diseases. NASA's Moderate Resolution Imaging Spectroradiometer (MODIS) instrument flown on the Terra and Aqua satellites has the temporal, spatial, and spectral properties to make it an excellent coarse-resolution data source for rapid, comprehensive surveillance of agricultural areas. A proof-of-concept wide area crop surveillance system using daily MODIS imagery was developed and tested on a set of San Joaquin cotton fields over a growing season. This area was chosen in part because excellent ground truth data were readily available. Preliminary results indicate that, at least in the southwestern part of the United States, near-daily NDVI products can be generated that show the natural variations in the crops as well as specific crop practices. Various filtering methods were evaluated and compared with standard MOD13 NDVI MODIS products. We observed that specific chemical applications that produce defoliation, which would have been missed using the standard 16-day product, were easily detectable with the filtered daily NDVI products.
\end{abstract}

Keywords-MODIS; NDVI time series; AVHRR; Cotton; Noise; Median Filter; Savitzky-Golay filter; Agriculture; Crop Diseases

\section{INTRODUCTION}

Effective response to crop disease outbreaks requires rapid identification and diagnosis of an event. An integrated, multiresolution, remote sensing monitoring system could be an effective way to identify rapid-onset changes to an area of agricultural production that may be indicative of a disease outbreak. We believe a crop observation system will consist of coarse-, medium-, and high-spatial-resolution imaging assets. Coarse-resolution systems (250-1000 m ground sample distance (GSD)) provide regional and continental views with the potential of high temporal revisits. These types of systems can help identify events that medium- and high-spatialresolution systems can then further investigate. Mediumresolution systems (10-100 m GSD) can provide detailed fieldlevel information, while high-spatial-resolution systems (<10 m GSD) can provide spatial analysis near the crop row level.

This work was funded by NASA SSC Center Director's Discretionary Fund. Participation in this work by Science Systems \& Applications, Inc., and by Computer Sciences Corporation was supported under NASA Task Order NNS04AB54T under the NASA Applied Sciences Directorate at the John C. Stennis Space Center, Mississippi.
Theoretically, this type of remotely sensed imagery could be the basis of a Crop Surveillance Decision Support System if the ground-based information, soil types, crop types, and meteorological data were combined and integrated in a Webbased geographic information system (GIS).

A near-daily vegetation index product, such as a Normalized Difference Vegetation Index (NDVI) at moderate spatial resolution, may serve as a good method for monitoring quick-acting diseases. NDVI is defined by the equation:

$$
N D V I=\frac{N I R-R E D}{N I R+R E D},
$$

where NIR and RED are the ground reflectances in the near infrared and red spectral bands. The NDVI values vary between -1.0 and 1.0. Changes in an NDVI value reflect changes in the vegetation condition; healthy vegetation absorbs visible radiation while reflecting NIR radiation. A NDVI high value is associated with healthy vegetation and near-full canopy.

However, producing a quality, daily NDVI product for crop surveillance purposes has significant challenges. First, only a few sets of satellites provide near-daily coverage. Also, the spatial resolution at which most satellites work is insufficient for field-level work. Another issue is that high quality NDVI products require the generation of reflectance maps that are based upon correcting solar geometry, earth-sun distance, aerosols, molecular scattering and absorption TOA radiance data [1]. Unfortunately most NDVI products are produced with minimal correction, usually limited to incorporating only geometry and earth-sun distance effects. These types of products are problematic for crop surveillance systems because they make it difficult to compare products generated by various remote sensing systems [2].

In general, it is most desirable to have high-quality reflectance maps that are atmospherically correct and account for all atmospheric effects. Even with atmospheric correction, NDVI products always contain variations associated with cloud contamination, incomplete atmospheric correction, and 
bidirectional reflectance effects. These variations can be considered a noise source because they are not correlated with real vegetation changes [3]. Typically, these types of noise sources are partially eliminated by a compositing method, where over a selected time only the maximum NDVI value is chosen. This approach, commonly called the maximum value composite method, is based on the assumption that cloudcontaminated pixels reduce the true NDVI value. Typical NDVI-composited time series applications include monitoring photosynthetic vegetative activity for phenologic and biophysical interpretations and change detection.

The problem with this approach is that the amount of time required to produce a composite (10 days or greater) is often significantly longer than the natural variation period in the crop. Consequently, critical events are missed, which is undesirable for a crop surveillance system.

NASA's Moderate Resolution Imaging Spectroradiometer (MODIS) instrument flown on the Terra and Aqua satellites has the temporal, spatial, and spectral properties to make it an excellent coarse-resolution first line of defense for potential rapid, comprehensive surveillance of our Nation's crops. MODIS has the potential of generating near-daily global NDVI at $250 \mathrm{~m}$ GSD. A significant advantage of MODIS over previous systems, such as the Advanced Very High Resolution Radiometer (AVHRR), is that MODIS provides higher spatial resolution imagery with better atmospheric correction. [5.]

In particular, MOD13 is a relevant MODIS product [4]. MOD13 is a comprehensive vegetation index product that includes an NDVI; this also complements AVHRR NDVI products. MOD13 includes a composited atmospherically corrected $250 \mathrm{~m}$ RED and NIR reflectance data produced on a 16-day cycle corrected for atmospheric and viewing geometry bidirectional reflectance distribution function (BRDF) effects. To evaluate and survey crop health, a 16-day period is too long, and higher temporal resolution is required to detect changes. The MOD13 product, however, is well validated and can be used to test other methods and other NDVI products.

\section{METHODS}

To address the high temporal requirement, we used the MOD09 daily $250 \mathrm{~m}$ reflectance product to create a daily NDVI [5]. MOD09 reflectance maps are generated daily using dark pixel atmospheric correction methods. The MODIS dark pixel atmospheric correction algorithm is based upon both locating dark pixels using the short-wave infrared (SWIR) bands and using visible bands to derive the aerosol properties. The reflectance in the SWIR band is generally highly correlated with visible bands and is less affected by atmospheric aerosols. The MODIS water detection bands are used to estimate water vapor. The derived atmospheric aerosols and water vapor are used in a radiative transfer model to generate atmospherically corrected products or reflectance maps.

We have developed a prototypical system, specifically for wide-area crop surveillance, that generates daily MODIS NDVI generation and display software. Using our custom software, we can display NDVI images from the MOD13 16-day NDVI product or from the daily NDVI calculated from the MOD09 reflectance product. The RED and NIR reflectance datasets, as well as the other datasets contained in these products, can also be directly displayed. Within the datasets, the region of interest can be subsetted by selecting either latitude and longitude ranges or row and column ranges. Additionally, after selecting a time period of interest, NDVI time series plots and image movies can also be created and displayed.

Temporal variations in the NDVI due to sensor, solar geometry, and atmospheric noise can be estimated for some closed-canopy configurations using least squares methods which can be used to set thresholds. Interpolation could/can be used to fill in values where cloud cover obscured the pixel. Interpolation is performed only in the temporal domain because spatial heterogeneity occurs at the pixel level and spatial interpolation will not reflect the true phenomena. Interpolation does require relatively small gaps in the temporal intervals between cloud-free days. This small gap requirement will not hold for many areas where the number of cloud-free days is limited. To reduce noise and to produce products similar in function to the MOD13 product, a median filter or a SavitzkyGolay (SG) filter can be applied to the interpolated data. SG filters are commonly used with noisy datasets to reduce noise while preserving peak shapes.

We evaluated the prototypical system by examining agricultural areas in the San Joaquin, CA, region and leveraging existing ground truthing data. Fig. 1 shows a shape file of the Sheely Farm fields obtained from the San Joaquin Valley NASA Ag20/20 program Web site [6]. This shape file shows the fields and the types of crops that were grown during the 2003 season: pistachios, two types of cotton, tomatoes, garlic, and wheat. Each field was/is assigned a numerical identification. This area was also chosen due to the large number of cloud-free days there; this allows an NDVI product to be produced every few days even without interpolation. This is highly desirable when performing research on natural crop variability. Small spatial subsets of this region were selected based upon computational memory constraints and crop treatments. Various MODIS products, over the growing season, were ordered from the Goddard Distributed Active Archive Center including MOD13, daily MOD09, and atmospheric products.

The MOD09 reflectance data was converted to an NDVI product using expression (1) and was compared with the standard MOD13 product for validation. The NDVI product was then transformed to a map projection so it could be easily integrated with a GIS to show other appropriate data, such as crop type, land cover, and meteorological data. Chemical applications for inducing flowering and defoliation were applied to a set of Sheely Farm cotton crop fields to serve as an analogue for a crop disease breakout. The fields were studied extensively with MODIS and other remote sensing assets to examine the change in NDVI associated with the defoliant application. Time series plots, image movies, interpolation, and noise analysis products were generated by the MODIS NDVI display software. 
Fig. 2 shows a $250 \mathrm{~m}$ GSD NDVI image created from a MOD09 data file of the Sheely Farm area. The outline of the Sheely Farm area of interest is plotted in red.

Using MOD09 or MOD13 data files of the same area over a period of time, we created/can create NDVI time series plots for a selected pixel. By selecting latitude and longitude ranges or by selecting row and column ranges, we also created/can create MOD09 or MOD13 image movies. We could/can choose to view raw NDVI data, interpolated data, or filtered data. Optionally, we could also/can fit a straight line to the data and calculate statistics related to noise.

Pixel metadata for the MOD09 and MOD13 products contain information on cloud conditions and on pixel quality. Pixels that the MOD09 and MOD13 algorithms were unable to calculate a valid pixel value are considered to be bad pixels. Bad pixels and pixels obscured by clouds are usually ignored. The missing points, or the pixel values that are considered less than ideal, can be interpolated temporally using a spline algorithm. Other interpolation algorithms may be more suitable for evaluating data containing large numbers of contiguous clouded pixels. To reduce noise, a median filter or an SG filter can be applied to the interpolated data. The SG filter requires two parameters: the polynomial order and the frame size. The polynomial order must be less than the frame size, and the frame size must be odd. An SG filter was selected for this software because of its robustness and its flexibility. Typically, median filters of three or five points have been used. A median filter has the advantage that the data need not be interpolated clouded pixels can simply be ignored. SG filters have been used successfully to smooth composited NDVI datasets [3].

\section{RESULTS}

An NDVI time series, using MOD09 data from the Aqua satellite, is shown in Fig. 3, along with the corresponding MOD13 NDVI. Because no interpolation or filtering was applied to the MOD09 NDVI, a significant amount of noise is apparent. Most of the very large, downward spikes are due to cloud cover. The pixel selected is in the 5-3 cotton field of Sheely Farm. Cotton was chosen because specific chemical applications can produce significant changes in canopy characteristics; because it goes to full canopy, the soilvegetation optical interactions become simplified. On 10/2/2003, 18 days before harvesting, a chemical preparation, which induced defoliation, was applied. Fig. 3 shows that the NDVI dropped drastically early in October; this corresponds to the time of chemical application. Use of the standard MOD13 16-day product alone would have delayed the detection of the chemical application.

For the same pixel, Fig. 4 shows a time plot of a 3-day median-filtered NDVI, resulting in significant improvements in the signal-to-noise ratio. Pixels obscured by clouds were ignored, resulting in elimination of many of the sharp downward spikes shown in Fig. 3. Similar results were obtained using an SG filter. In general, the daily NDVI products followed the MOD13 trends; however, the daily NDVI time series appears to better follow the natural variations in crops. Some pixel values in other nearby cotton fields were much noisier over the same period of time. In particular, field
31-3 showed peak-to-peak NDVI variations approximately 0.1 compared to approximately 0.5 for field 53 . Some of the other cotton fields showed a more gradual change in NDVI over several days after the chemical application.

Because the filtering noise reduction shown in Fig. 4 is large, we systematically analyzed the noise in the NDVI data for cotton fields. To analyze the noise, we fit a straight line, $y=A+B x$, to the NDVI data for the periods where the crop characteristics changed slowly. We then calculate the standard deviations $(\sigma)$ in the slope, $B$, in the y-intercept, $A$, and in $y$ itself. Fig. 5 shows a time plot of the NDVI of a pixel in the 31-3 cotton field. The y-intercept and slope of the straight line are shown at the top, and the standard deviations in $y, A$, and $B$ are also shown. Based on the pixel metadata, clouded pixels were ignored. With no filtering applied, $\sigma_{y}$ is approximately 0.1. Fig. 6 shows the results using a 3-day median filter. The median filter reduced $\sigma_{y}$ by approximately 40 percent. Using the SG filter resulted in very similar results but in some cases caused some artificial ringing in the filtered products. Similar improvements were found upon examination of several other fields in the Sheely Farm region.

During this demonstration effort, we found that a near-daily set of crop observations is necessary for crop monitoring. We found that some phenomena can be observed within 5 days after an event, such as a chemical defoliant application; however, we also observed that the 16-day product completely missed the early onset of plant defoliation.

Variability in time series between different pixels over a given field was generally very small. We did see significant variability between different cotton fields that cannot be attributed to sensor or atmospheric noise and may be associated with the practices used at that given field.

For many fields, we found that the $250 \mathrm{~m}$ product is sufficient to resolve individual fields, and we found similar results for several pixels over a given field. In some cases, larger pixels would have mixed crop types.

Smoothing of MODIS-derived time series to improve the signal-to-noise ratio associated with atmospheric and BRDF effects is a research area that is still developing. Without compositing, a large variation in individual daily responses can occur. In some the work presented here, digital filtering reduced the noise by a factor of nearly two while having minimal impact on the phenomena of interest. In cases with a significant percentage of cloudy days, the approaches described here may not be as effective. Perhaps combining data from multiple satellites and sensors could help, however, these systems would/will be need to be normalized to some common scale. Spatial resolution differences between systems would/will also have to be taken into consideration

Although a wide range of diseases and pathogens have been identified, their spectral, temporal, and spatial signatures are not well described. The tools developed during our study could be valuable for such research. Due to the area's low percentage of cloud cover, the southwestern part of the United States could/can serve as laboratories for determining the expected natural variations in a vegetation canopy. 


\section{ACKNOWLEDGMENTS}

The authors thank Marcia Wise for her careful editing of this manuscript. We thank Matt Bethel of the Institute for Technology Development for the shape files and ground truth data for the Sheely farm. We also thank Randy Greer and Jerry Gasser of Lockheed Martin Space Operations for their programming support.

\section{REFERENCES}

[1] P.-Y. Chen, R. Srinivasan, G. Fedosejevs, and J.R. Kiniry, "Evaluating different NDVI composite techniques using NOAA-14 AVHRR data," Int. J. Remote Sens., vol. 24, no. 17, pp. 3403-3412, September 2003.

[2] M. Pagnutti, K. Holekamp, R.E. Ryan, R. Vaughan, J. Russell, and D. Prados, "Atmospheric correction of high spatial resolution commercial satellite imagery products using MODIS atmospheric products,” (This Document), Proceedings of the Third International Workshop on the Analysis of Multitemporal Remote Sensing Images, Biloxi, MS, 16-18 May 2005.

[3] J. Chen, P. Jönsson, M. Tamura, Z. Gu, B. Matsushita, and L. Eklundh, "A simple method for reconstructing a high-quality NDVI time-series data set based on the Savitzky-Golay filter," Remote Sens. Environ., vol. 91, issues 3-4, pp. 332-344, June 2004.

[4] A. Huete, C. Justice, and W. van Leeuwen, MODIS Vegetation Index (MOD 13): Algorithm Theoretical Basis Document. Version 3. April 30, 1999, $131 \mathrm{p}$. http://eospso.gsfc.nasa.gov/ftp_ATBD/REVIEW/MODIS/ATBD-MOD13/atbd-mod-13.pdf, accessed January 2005.

[5] E.F. Vermote and A. Vermeulen, Algorithm Technical Background Document: Atmospheric correction algorithm: spectral reflectances. Version 4.0, MODIS Product ID: MOD09,April, 1999. http://modis.gsfc.nasa.gov/data/atbd/atbd_mod08.pdf .

[6] Opto-Knowledge Systems Inc., 2003 Remote Sensing Data: Ag20/20 Precision Agriculture Activities in the California San Joaquin Valley. National Aeronautics and Space Administration; UC Davis, CSTARS; USDA/ARS. http://www.ag2020.net, accessed March 2005.

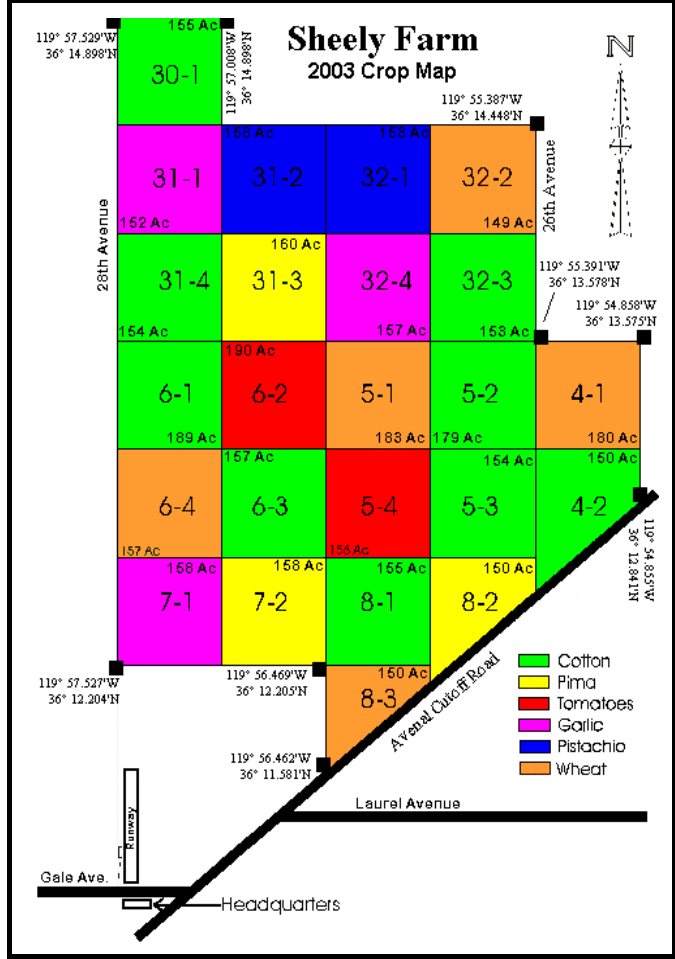

Figure 1. Sheely Farm 2003 Crop Map shape file, San Joaquin, CA.

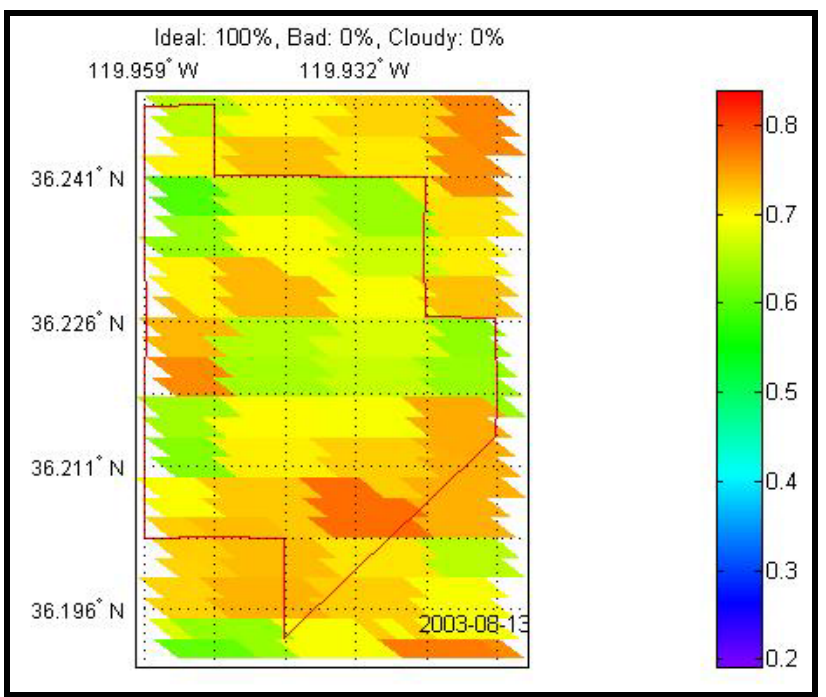

Figure 2. NDVI calculated from MOD09 dataset over the Sheely Farm. 


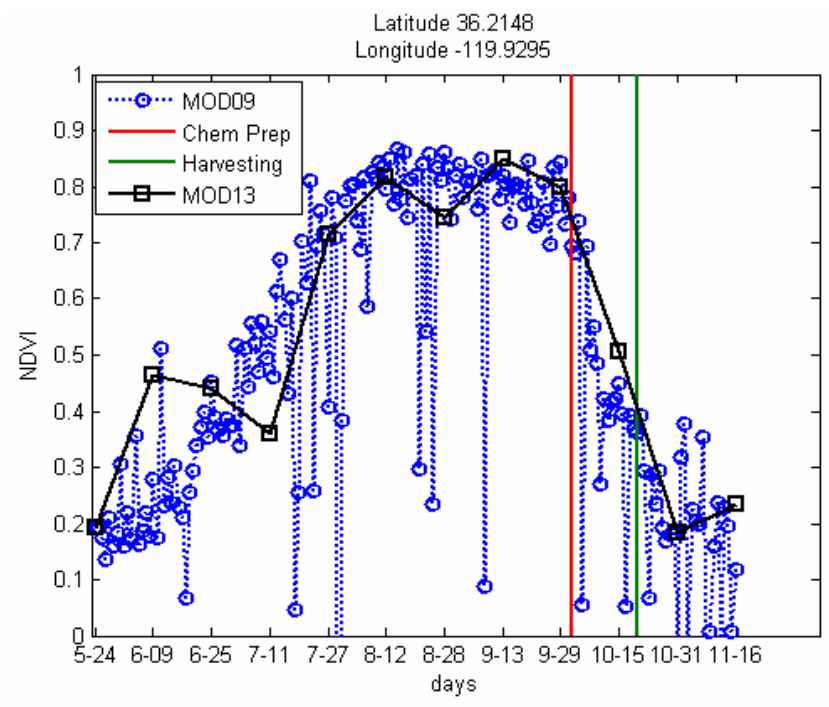

Figure 3. Comparison of raw MOD09 and MOD13 imagery for the 5-3 cotton field.

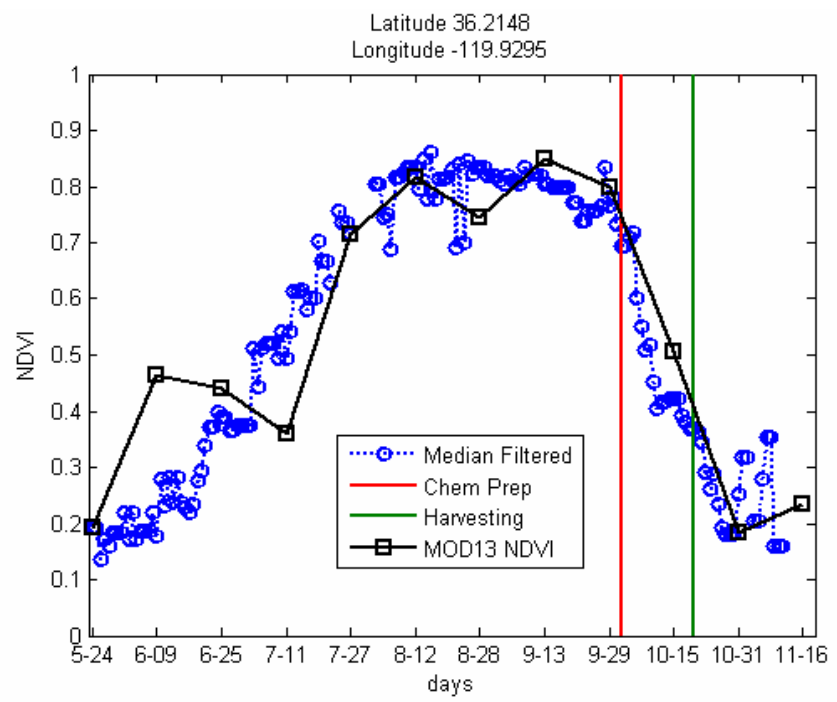

Figure 4. Median-filtered NDVI for field 5-3, ignoring pixels affected by clouds.

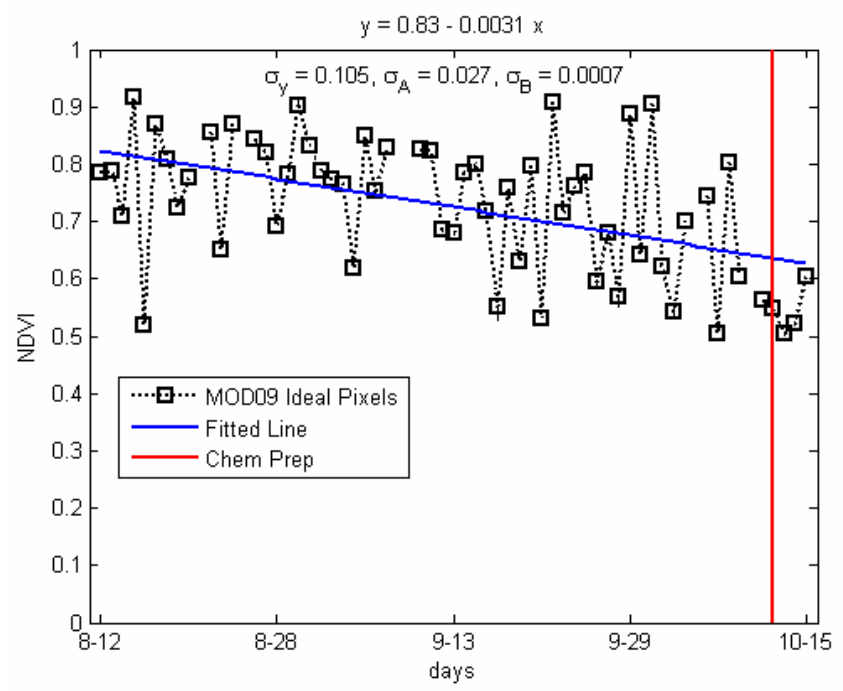

Figure 5. Time plot for a pixel in field 31-3 of Sheely Farm with no filtering applied to the NDVI data.

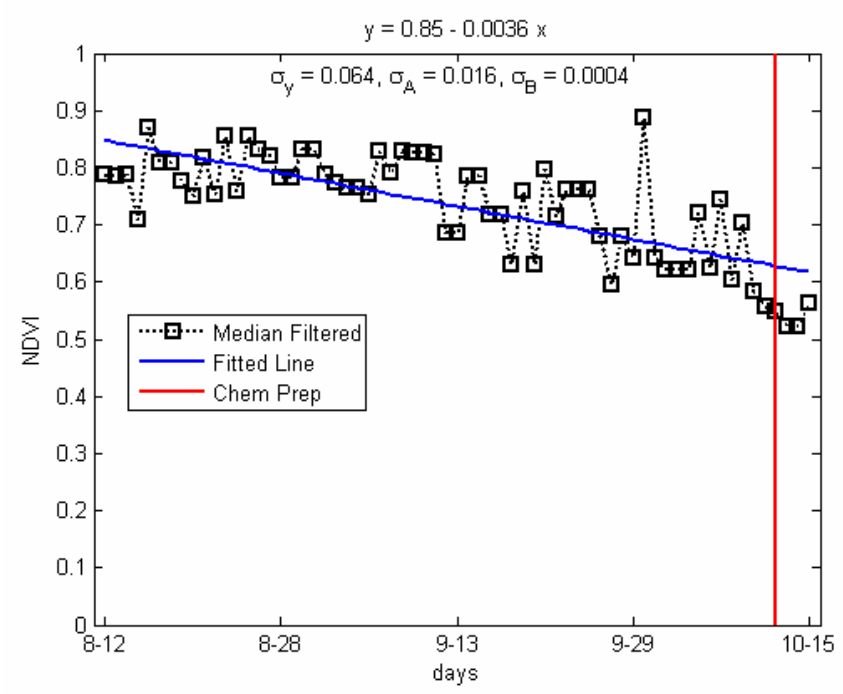

Figure 6. Time plot for a pixel in field 31-3 of Sheely Farm with a 3-day median filter applied to the NDVI data. 